\title{
Eggerthella lenta
}

National Cancer Institute

\section{Source}

National Cancer Institute. Eggerthella lenta. NCI Thesaurus. Code C86349.

A species of aerobic, Gram positive, rod shaped bacterium in the phylum Actinobacteria.

This species is non-fermenting, liquefies gelatin, reduces nitrate, does not hydrolyzes

esculin or starch, nonhemolytic and catalase negative. E. lenta is a commensal organism of the gastrointestinal tract and is rarely associated with infections. 